\title{
TSPO-targeted agents for PDT of aggressive tumors (Withdrawal Notice)
}

Meng Su, Qing Xie, Dawei Zhang, Mingfeng Bai

Meng Su, Qing Xie, Dawei Zhang, Mingfeng Bai, "TSPO-targeted agents for PDT of aggressive tumors (Withdrawal Notice)," Proc. SPIE 11070, 17th International Photodynamic Association World Congress, 110701S (7 August 2019); doi: 10.1117/12.2525334

SPIE Event: 17th International Photodynamic Association World Congress, 2019, Cambridge, Massachusetts, United States 


\section{TSPO-targeted agents for PDT of aggressive tumors (Withdrawal Notice)}

Meng Su, Qing Xie, Dawei Zhang, Mingfeng Bai

Vanderbilt Univ. Institute of Imaging Science (United States)

Proc. SPIE 11070, 110701S (2019)

Online Publication Date: 7 August 2019

Withdrawn from Publication: 12 August 2019

Conference Date: 28 June-4 July 2019

Conference Location: Cambridge, Massachusetts, United States

Conference Title: 17th International Photodynamic Association World Congress

Conference Chairs: Tayyaba Hasan

Publisher's Note: This paper, originally published on 7 August 2019, was withdrawn at the request of the authors on 12 August 2019. 